\title{
Optimal Outcomes for Oropharyngeal Dysphagia
}

\author{
Jeri A. Logemann • Laura L. Pitts
}

Published online: 9 October 2013

(c) Springer Science + Business Media New York 2013

\begin{abstract}
Returning a patient with oropharyngeal dysphagia to a full, safe oral intake of a normal diet is optimal, but not always possible. With intervention, other optimal outcomes may exist along the patient's specific trajectory of recovery or degeneration. The measurement points along the maximized trajectory of recovery through applied intervention are then patient-specific optimal outcomes. Measurement tools for dysphagia must be valid, reliable, and sensitive to diverse populations and capture clinically meaningful change. We suggest distinctive levels of quality for outcome measurement tools, and call for further research to determine appropriate outcome measures and meaningful end points in dysphagia management. The conceptual framework provided regarding optimal outcomes in dysphagia management can facilitate discussions regarding health care reform. Large clinical data sets and future research are needed to better define the trajectory of swallowing rehabilitation, a significant and positive deviation from the natural disease or illness course.
\end{abstract}

\section{J. A. Logemann $(\bowtie)$}

Communication Science and Disorders, Otolaryngology-Head \&

Neck Surgery and Neurology, Northwestern University,

Frances Searle Building, 2240 Campus Drive, Room 3-358,

Evanston, IL 60208-3507, USA

e-mail: j-logemann@northwestern.edu

L. L. Pitts

Rehabilitation Institute of Chicago, 345 E. Superior Street,

Suite 1353A, Chicago, IL 60611, USA

e-mail: 1pitts@ric.org

L. L. Pitts

Physical Medicine and Rehabilitation, Feinberg School of Medicine, Northwestern University, Chicago, IL 606011, USA
Keywords Dysphagia - Outcome measures - Optimal outcomes · End points - Aging effects - Degenerative disease $\cdot$ Acute onset dysphagia

\section{Introduction}

The optimal outcome for oropharyngeal dysphagia management is returning the patient to full, safe oral intake on a normal diet as quickly as possible. However, there are so many anatomic and/or physiologic abnormalities associated with oropharyngeal dysphagia that this optimal outcome is not always possible. The purpose of this article is to (1) conceptualize the influence of etiology on optimal outcomes, (2) suggest distinctive levels of quality for outcome measures, and (3) emphasize the need for further research to select appropriate outcome measures and establish meaningful end points in dysphagia management.

\section{Cause and Optimal Outcomes for Oropharyngeal Dysphagia}

We propose a tiered framework for conceptualizing optimal outcomes relative to three general types of dysphagia: (1) acute onset, (2) progressive, and (3) age-related dysphagia. Certainly, the cause of oropharyngeal dysphagia is not the sole determinant of optimal outcomes; however, etiology is included here as a key factor [1•].

Acute Onset Dysphagia

The causes of oropharyngeal dysphagia include sudden onset disorders such as stroke, head injury, spinal cord injury, and surgical procedures for head and neck cancer 
from which rehabilitation, including return to a normal or near-normal swallow, is possible [2, 3]. At times acute medical conditions such as, multiple strokes or medullary/ pontine strokes are severe enough that return to normal swallow function is not always possible [4, 5]. Nevertheless, spontaneous recovery in some acute patients can considerably facilitate recovery; however, more data defining the extent intervention can maximize the slope of recovery are critical [6]. Other acute causes of oropharyngeal dysphagia, including neuromuscular diesase such as Guillain-Barré syndrome may create significant oropharyngeal swallow impairment $[1 \bullet, 7,8]$.

\section{Progressive Dysphagia}

Almost all progressive neurologic diseases cause dysphagia during their time course and that dysphagia usually progressively worsens. The optimal outcomes for this group of patients are quite different from the outcomes for sudden onset disorders. The optimal outcome as these diseases progress will evolve into maintaining oral intake, perhaps with some limitations on the diet or food consistencies that the patient can manage [9]. Regular follow-up with the patient is critical to introduce appropriate intervention and maintain oral intake throughout the disease process [10••].

Chemoradiotherapy to the head and neck can also significantly and progressively impair oropharyngeal swallow function. The severity of the swallow problems will depend on the radiation dose to the region as well as the way in which chemotherapy is used, either in sequence or concurrently with radiotherapy $[11,12]$. Although the surgical patient may have lost anatomic structure, the muscles and other tissues that remain are normal and better able to respond well to exercise therapy [13]. In contrast, the patient with chemoradiotherapy has no structural alterations, but has muscle tissues that have lost some blood supply (one effect of radiotherapy). In the case of concurrent chemotherapy and radiotherapy, the chemotherapy actually intensifies the effect of the radiotherapy, resulting in fibrosis. This can leave the patient unable to swallow over the years after treatment and perhaps make swallowing therapy less effective. Because the change in muscle fibers to a fibrous state happens gradually, it may be a number of years before patients recognize that their swallowing has deteriorated and they are unable to eat a normal diet. By this point, return to normal oral intake is highly unlikely, and the most optimal outcome at that time may be maintaining adequate oral intake on a reduced or restricted diet [14].

\section{Age-Related Dysphagia}

Another general type of oropharyngeal dysphagia can emerge as a consequence of normal aging in combination with a temporary milder disorder such as a urinary tract infection or influenza [1, 15]. Normal aging creates changes in the oropharyngeal swallow (i.e., presbyphagia), which alone are usually not enough to create dysphagia, but they increase the risk of dysphagia during times of illness. Normal healthy aging results in systematic and predictable changes in the normal oropharyngeal swallow which typically begin between 50 and 60 years of age [16, $17 \bullet \bullet, 18]$.

Specifically in normal aging, oral transit time and triggering of the pharyngeal swallow tends to slow to 1-2 s, and penetration into the airway becomes more frequent, but not necessarily aspiration [16, 17••]. The extent of hyolaryngeal elevation and anterior movement, the major contributor to opening the upper esophageal sphincter, is also generally mildly reduced, especially in older men [19•]. With age, men in particular demonstrate a reduction in muscular reserve, which negatively affects the speed and range of motion of oral and pharyngeal structures [19•, 20]. The range of motion of pharyngeal structures involved in the swallow such as hyoid and laryngeal movement may be functioning adequately when the patient is healthy, but may become pathologically reduced with illness. Unfortunately, loss of functional reserve is increased when older patients are bed-bound because of illness [21, 22].

When the patient becomes ill and muscular reserve is further reduced, the muscle movements may no longer be adequate to produce a normal swallow. For this reason, swallowing clinicians must be aware that the elderly patient who is hospitalized should receive physical therapy in order to maintain normal muscle function for appendicular and axial functions [23]. Normal elderly individuals who have the compounding effects of aging plus illness and were eating a normal diet safely before their illness should ideally be returned to their prior safe oral intake. It is important that swallowing clinicians recognize the differences in optimal outcomes for each of these different types of oropharyngeal dysphagia.

One limitation in defining optimal outcomes for oropharyngeal dysphagia across all patients is our lack of knowledge of the exact trajectory of recovery (TOR) for each medical diagnosis: specifically, the time points for clinically meaningful change. For the purposes of this article, the TOR can be defined for either the natural disease course without intervention [i.e., trajectory of nature (TON)] or the redefined TOR through intervention [trajectory of intervention (TOI)]. Without being able to anticipate changes that will occur in a patient with a particular cause of oropharyngeal dysphagia, the swallowing therapist will always be "behind the curve" relative to how to prepare the patient for what may be coming and to provide timely reassessment and treatment. 
Table 1 Optimal outcomes by generalized trajectory of recovery

\begin{tabular}{llll}
\hline & Generalized trajectory of recovery & & \\
\cline { 2 - 4 } & Acute onset & Progressive & Age-related \\
\hline $\begin{array}{c}\text { TOI level 1: } \\
\text { nonrestrictive }\end{array}$ & $\begin{array}{c}\text { Safe intake of a full oral diet. } \\
\text { Further management is not } \\
\text { needed unless there is a change } \\
\text { in medical status }\end{array}$ & $\begin{array}{c}\text { Safe intake of a full oral diet or } \\
\text { postponement of diet reduction. } \\
\text { Continual management as disease/ } \\
\text { disorder progresses }\end{array}$ & $\begin{array}{c}\text { Safe intake of a full diet as appropriate to } \\
\text { peers. Prevention or management as needed } \\
\text { with change in medical status beyond } \\
\text { normal aging }\end{array}$ \\
$\begin{array}{c}\text { TOI level 2: } \\
\text { compensation }\end{array}$ & $\begin{array}{c}\text { Oral diet with compensation and } \\
\text { management for diet upgrade } \\
\text { with medical status }\end{array}$ & $\begin{array}{c}\text { Oral diet with compensation. } \\
\text { Continual management for nutrition } \\
\text { and quality of life }\end{array}$ & $\begin{array}{c}\text { Oral diet with compensation with continual } \\
\text { management for diet upgrade to the peer } \\
\text { level and nutritional/quality-of-life }\end{array}$ \\
$\begin{array}{c}\text { TOI level 3: } \\
\text { supplementation }\end{array}$ & $\begin{array}{c}\text { Partial oral diet with management } \\
\text { for diet upgrade with medical } \\
\text { status improvement }\end{array}$ & $\begin{array}{c}\text { Partial oral diet with continual } \\
\text { management for nutrition and } \\
\text { quality of life }\end{array}$ & $\begin{array}{c}\text { Partial oral diet with continual management } \\
\text { for diet upgrade closer to the peer level and } \\
\text { nutrition/quality of life }\end{array}$ \\
$\begin{array}{c}\text { TOI level 4: lack } \\
\text { of positive } \\
\text { response to } \\
\text { intervention }\end{array}$ & $\begin{array}{c}\text { The appropriate patient receives adequately trialed and judicious intervention and is discharged from intervention secondary } \\
\text { to lack of positive response (e.g., } \\
\text { severe medullary stroke, advanced Alzheimer's disease) }\end{array}$ \\
\hline
\end{tabular}

TOI trajectory of intervention

To illustrate optimal outcomes in regard to the three general types of dysphagia, we suggest a theoretical framework of three different TOIs with associated levels of optimal outcomes (Table 1). The appropriate level of optimal outcomes for a patient may be determined by additional prognostic factors (e.g., insult or disease severity/cause, comorbidities, cognitive status, medical stability, fatigue, repair-related neurofactors, and even genetics), access to care, and the patient's response to intervention [1]. Research continues to identify and define to what extent patient and environmental factors define the TOI at an individual case level. All optimal outcomes also strive for the prevention or postponement of deleterious, dysphagia-related outcomes such as aspiration pneumonia and hospital readmission at incidence levels equal to or better than those for the general population.

Within this framework, optimal outcomes in TOI levels 1-3 would be projected for patients who respond positively to intervention. In contrast, level 4 exemplifies the case in which the best possible outcome is a thoroughly trialed intervention with documented lack of positive response to the intervention. Despite a lack of positive response, a meticulous and careful trial of swallowing intervention is favorable compared with not attempting intervention for an appropriate patient. Additionally, all patients with level 4 outcomes should be reevaluated for future response to intervention and case management as needed [1].

The current pulse of dysphagia rehabilitation reimbursement policy has produced escalating pressure to measure clinical outcomes from a quality perspective. This is complicated by the myriad of optimal outcomes across dysphagia types illustrated in Table 1. As policy interests are directed toward identifying quality indicators to determine value-based payments, a critical concern for dysphagia services is to establish valid and reliable metrics that demonstrate their value. Since all dysphagic patients do not follow a standardized, positively directed linear course to a full oral diet, the value of our assessment and treatment is reflected in our ability to efficiently maximize the magnitude of recovery toward a positive and optimal trajectory.

Therefore, the therapeutic TOI must demonstrate a departure from the natural disease or injury course (TON) in both functional gain, rate of recovery or even through a reduction in decline. Significant and meaningful improvement in function would be evident in superimposing the disease course with the individual's intervention-induced performance; the TOI would theoretically display a positive change in trajectory (i.e., change in direction, slope, or level of recovery) compared with the TON. Optimal outcomes could then be theorized as end points along the maximized TOI.

Figure 1 illustrates this concept by plotting the projected TOI and TON for each of the three dysphagia types: acute onset, progressive, and age-related dysphagia. In each graph, three hypothetical TOIs, one for each level of optimal outcomes, are represented at two time points during intervention. Level 4 is not charted as the TOI of level 4 would not differ from the TON owing to lack of positive response to intervention. The TOI and TON are plotted in regard to diet with end points matching those described in Table 1. The vertical axis quantifies diet using the sevenpoint functional oral intake scale (FOIS) [24] with diet classifications 1-3 referring to tube dependency with or without oral intake. FOIS scores 4-7 represent an increasing variety of total oral intake with increasing 
scores. Level 7 represents a total oral diet without restrictions or compensations.

Specifically, a level 1 optimal end point for acute onset dysphagia is to achieve a full diet without compensation or supplementation. A level 2 end point would be to achieve full oral intake with compensation (FOIS level 1-6), and a level 3 end point may include increased oral intake with concurrent enteral feeding at a rate better than that expected with spontaneous recovery (FOIS level 1-3) with continual management for progression toward a completely oral diet.

In contrast, an optimal level 1 outcome for progressive dysphagia maintains or increases diet levels in the direction opposite to the TON. Level 2 reduces the decline in swallowing function and maintains full oral intake (FOIS level from 6 to 4 ). Even level 3 demonstrates a positive response to intervention by reducing the negative slope of the TON to an optimal end point of partial oral intake (FOIS level from 6 to 3 as opposed to FOIS level from 6 to 1 ).

Natural aging in healthy adults is illustrated in Fig. 1 as an FOIS level of 7 with possible further age-related change in function over time (i.e., illustrated by the dotted line). A departure from the full oral diet without restriction occurred because of illness (represented by the downwardpointing arrow) to an FOIS level of 3. Therefore, from the reduced function (FOIS level 3), aging effects would be superimposed on the TON and may influence function over time. Optimal outcomes may include the prevention of further age-related decline (level 3), an increase to full oral diet with compensation (level 2), or a return of swallow to normal, peer-level performance or even exceeding it (level 1). Additionally, a level 1 TOI may encompass preventative treatment to reduce an imposing slope of natural aging and maintain function.

\section{Quality Measures are Necessary to Document Optimal Outcomes}

The FOIS level is applied here as an outcome measure to conceptualize possible deviations of the TOI from the TON in regard to diet change, all of which represent a positive, optimal response to intervention. In isolation, diet change has been established as a problematic outcome measure as noncompliant patients may advance their diet despite persistent physiological impairment and professional recommendations $[25 \bullet \cdot, 26]$. Furthermore, diet recommendations are associated with factors beyond physiological function, including patient preference, clinical ethics, and end-of-life nutrition decisions. Researchers and clinicians are presently engaged in discussions regarding the establishment and evaluation of quality outcome measures for dysphagia rehabilitation across health care settings.
Multiple systematic reviews report that the effectiveness of therapeutic approaches is heavily dependent on the outcome measurement tool (e.g., instrumental, patientreported outcomes, clinical observation, or patient health status) and the evaluation protocol (e.g., number of swallowing trials, bolus volume, and food consistency) [27, 28]. Optimal outcomes depend on a careful and accurate evaluation that determines the disordered physiology in the patient's swallow. Clinical evaluation measures may be used as outcome measures to reduce redundancy and burden if they can demonstrate (1) sufficient reliability across settings, (2) sensitivity to change across populations and the timeline of care, (3) cost-effectiveness, (4) the ability to capture clinically significant change within functional areas about which the population is concerned and (5) are repeatable.

\section{Dysphagia Screen and Clinical Swallowing Evaluation}

A screen is the first defense in identifying patients with either significant or insignificant risk of dysphagia and in determining the need to complete a dysphagia evaluation. It is a pass/fail procedure that must be short and noninvasive, as the cost is not reimbursed by third-party payers. Therefore, a screening procedure by definition is not an outcome measure for treatment, because of its dichotomous nature and the fact that it is only administered prior to formal evaluation. In contrast, a clinical swallowing evaluation is a behavioral assessment of oral anatomy and physiology including inferred judgment of select pharyngeal and laryngeal function during swallowing.

\section{Instrumental Evaluation}

Videofluoroscopy is the commonest instrumental outcome measure for randomized clinical trials of oropharyngeal dysphagia therapy [27]. Preliminary evidence also suggests its cost-effectiveness by reducing direct medical costs associated with pneumonia [29]. After defining the patient's swallowing physiology, introducing treatment techniques during videofluoroscopic evaluations including postural changes [30], heightening sensory input prior to the swallow [31], swallow maneuvers, and diet change $[10 \bullet \bullet, 32,33]$, it is critical to quickly achieve optimal dysphagia outcomes for the patient. This can be difficult in situations where instrumentation, such as that used in videofluoroscopy, is not available.

In nursing homes and in situations where patients are cared for at home, it can be difficult to obtain access to an instrumental diagnosis. Referral of patients to a swallowing clinician with experience and access to instrumental assessment can be important in achieving optimal outcomes. Physiologic assessement and experienced clinicians 
Fig. 1 Comparative trajectory of intervention (TOI) for acute onset, progressive, and agerelated dysphagia. This concept is illustrated by plotting the projected TOI and trajectory of nature $(T O N)$ for each of the three dysphagia types: a acute onset, $\mathbf{b}$ progressive, and $\mathbf{c}$ agerelated dysphagia. FOIS functional oral intake scale
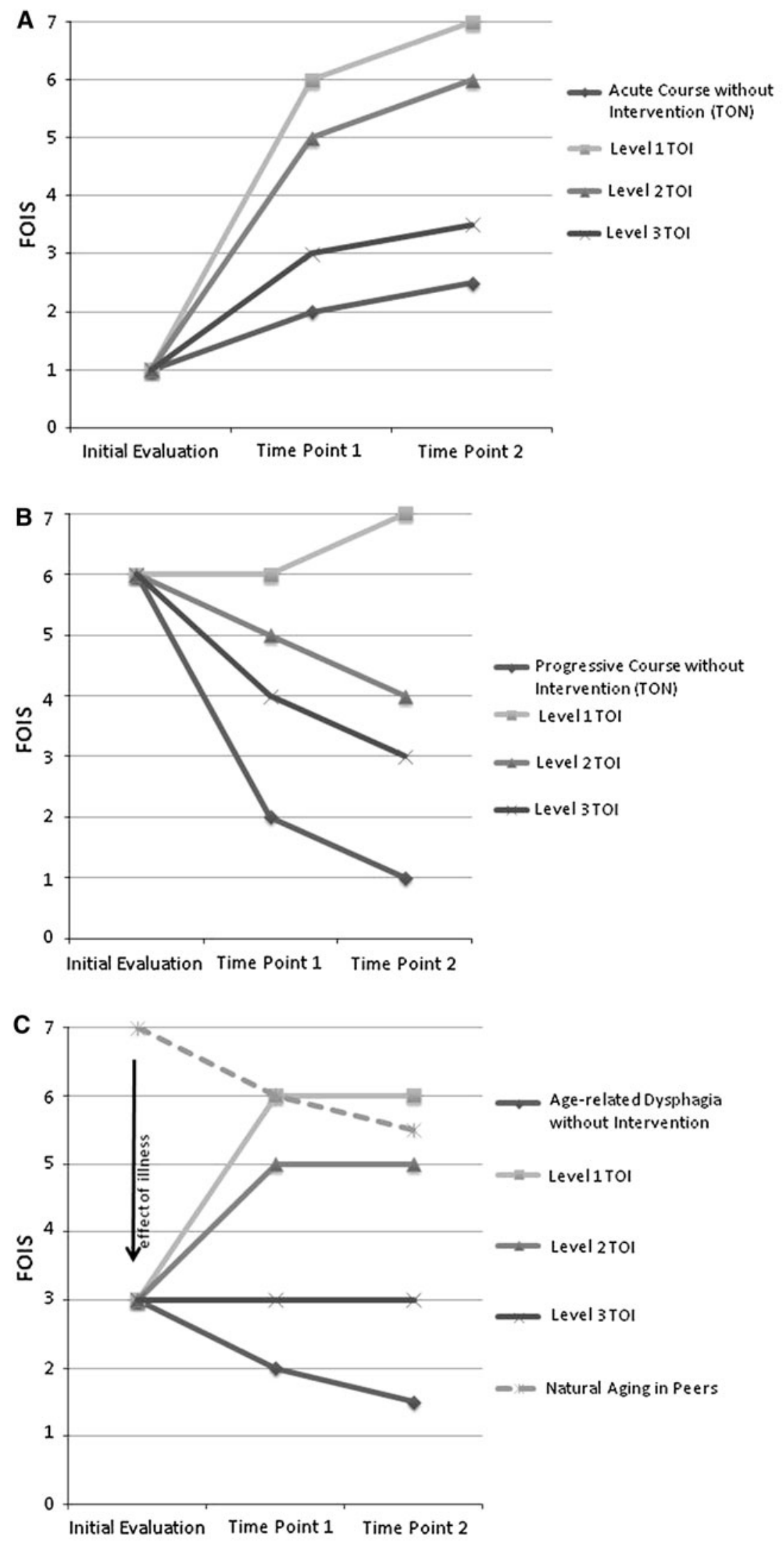
in the management of oropharyngeal dysphagia will provide the patient with the most accurate evaluation and optimal treatment plan. There are, however, a growing number of portable videofluoroscopy machines that travel in vans to these locations to evaluate patients with dysphagia. This can improve the situation of patients cared for at home or in a nursing facility with reduced access to instrumental assessment.

Alternatives to videofluoroscopic evaluation include the fiber-optic endoscopic evaluation of swallowing and even real-time magnetic resonance imaging [34, 35]. Fiber-optic endoscopic evaluation of swallowing permits visualization of the hypopharynx and larynx without exposure to radiation, with excellent safety; however, no information is gained about the physiology of the oral phase or during the swallow. Real-time magnetic resonance imaging provides visualization of the swallow anatomy and physiology across all phases of the swallow; however, this occurs at considerable cost and patient compliance.

Other objective measurement techniques to define swallowing mechanics have been advocated, and include manometry, lingual-palatal pressures, and external hyolaryngeal elevation/excursion. These measures are removed, however, from direct visualization of the swallow. Therefore, in regard to quality metrics, these measures must be strengthened by established normative data and/or validation with the established assessment procedures that allow direct visualization.

\section{Patient-Reported Outcomes}

Historically, outcome measures for dysphagia have been based on eradication of aspiration or improved biomechanical function and diet; however, there has been increased interest, specifically from scientific review committees and the Centers for Medicare and Medicaid Services, in the analysis of patient perception of quality of life and treatment outcomes [36]. Patient-reported outcomes may include questionnaires, visual analog scales, or Likert scales completed by patients. These measures will not be accurate or reliable in the presence of cognitive impairment, oropharyngeal sensory deficits, therapeutic misconception, and reporting biases or with populations with whom the instrument has not been validated.

Owing to the variety of direct and indirect swallowing measurement tools, a framework is proposed which suggests that the further removed the measure is from direct instrumental visualization, the greater the clinical inference regarding swallowing physiology (Table 2). Therefore, quality levels of measurement may exist depending on how removed these measures are from direct visualization and their established reliability, validity, sensitivity, and specificity.
Table 2 Description of quality levels of measurement

\begin{tabular}{|c|c|c|}
\hline $\begin{array}{l}\text { Quality levels of } \\
\text { measurement }\end{array}$ & Description & Examples \\
\hline \multirow{3}{*}{$\begin{array}{l}\text { Level 1: direct } \\
\text { visualization of } \\
\text { swallowing } \\
\text { physiology }\end{array}$} & \multirow{3}{*}{$\begin{array}{l}\text { Reliable and valid } \\
\text { instrumental tools } \\
\text { that permit } \\
\text { visualization and } \\
\text { quantification of } \\
\text { internal swallowing } \\
\text { anatomy and } \\
\text { physiology }\end{array}$} & $\begin{array}{l}\text { VFSS: visualization } \\
\text { of before, after, and } \\
\text { during the swallow }\end{array}$ \\
\hline & & $\begin{array}{l}\text { FEES: visualization } \\
\text { of before and after } \\
\text { the swallow as well } \\
\text { as vocal fold/ } \\
\text { laryngeal anatomy } \\
\text { and physiology }\end{array}$ \\
\hline & & $\begin{array}{l}\text { Real-time MRI: } \\
\text { visualization of } \\
\text { before, after, and } \\
\text { during the swallow }\end{array}$ \\
\hline \multirow[t]{2}{*}{$\begin{array}{l}\text { Level 2: validated } \\
\text { against direct } \\
\text { visualization of } \\
\text { swallowing } \\
\text { physiology }\end{array}$} & $\begin{array}{l}\text { Instrumental measures } \\
\text { without direct } \\
\text { visualization and } \\
\text { therefore require } \\
\text { inference via } \\
\text { established validity } \\
\text { against level } 1 \text { data } \\
\text { or normative data }\end{array}$ & $\begin{array}{l}\text { FEES: inference to } \\
\text { physiology during } \\
\text { the swallow }\end{array}$ \\
\hline & $\begin{array}{l}\text { Validated, reliable } \\
\text { PRO measures for } \\
\text { the patient } \\
\text { population or } \\
\text { family/caregiver. } \\
\text { The reporter must } \\
\text { demonstrate } \\
\text { appropriate } \\
\text { cognition, and } \\
\text { awareness }\end{array}$ & $\begin{array}{l}\text { Biokinematic } \\
\text { measures for } \\
\text { swallowing with } \\
\text { established norms }\end{array}$ \\
\hline \multirow[t]{2}{*}{$\begin{array}{l}\text { Level 3: not } \\
\text { validated against } \\
\text { direct } \\
\text { visualization of } \\
\text { swallowing } \\
\text { physiology }\end{array}$} & $\begin{array}{l}\text { Measures applied to } \\
\text { populations without } \\
\text { validation. } \\
\text { Observations at the } \\
\text { clinical bedside } \\
\text { evaluation without } \\
\text { norms or level } 1 \text { data }\end{array}$ & $\begin{array}{l}\text { Clinical observation } \\
\text { or measurement for } \\
\text { each unique case }\end{array}$ \\
\hline & $\begin{array}{l}\text { PRO measures not } \\
\text { validated with level } \\
1 \text { measures }\end{array}$ & $\begin{array}{l}\text { PROs of patient } \\
\text { groups with } \\
\text { variable cognition } \\
\text { or without } \\
\text { validation to level } 1 \\
\text { measurement }\end{array}$ \\
\hline
\end{tabular}

FEES fiber-optic endoscopic evaluation of swallowing, MRI magnetic resonance imaging, $P R O$ patient-reported outcome, VFSS videofluoroscopic swallowing study

\section{Timing of Optimal End Points}

Currently, complete data are not available to guide clinicians and third-party payers regarding the timing one can expect for various types of dysphagic patients to achieve optimal oropharyngeal dysphagia outcomes. Finding the most effective treatment plan for a patient requires both a 
careful and an in-depth evaluation of the oropharyngeal swallow and a good understanding of the patient's cognitive ability, motivation, available family support, and caregivers [37]. The time it takes to achieve optimal outcomes may depend on these factors as well as the nature and severity of the patient's oropharyngeal swallow disorders. Finally, choosing end points of intervention may be best defined by and specific to the TOR. For example, stroke recovery may experience different TORs during early, chronic, and late chronic phases [38].

\section{Conclusion}

In this article, we have taken both a pragmatic and theoretic approach to defining optimal outcomes for oropharyngeal dysphagia. The number of causes and severities of oropharyngeal dysphagia are vast, but we have categorized these into three groups: (1) acute onset, (2) progressive damage or disease, and (3) the interaction of aging and illness.

Theoretically, we have defined TOI for these three groups of patients. We conceive that intervention for oropharyngeal dysphagia can significantly impact the trajectory of swallowing recovery even when the therapeutic trajectory is overlaid on disease progression or spontaneous recovery. We have emphasized the importance of physiologic assessment of the oropharyngeal swallow, including the assessment of intervention and its effectiveness during visualization of swallowing physiology.

The proposed levels of TOR and the quality levels for measurement tools are simple frameworks to further the discussion on how to determine and measure optimal outcomes in dysphagia management. These outlines are purely conceptual, in the hope that they can facilitate dialogue among researchers, clinicians, health care networks, and third-party payers [39]. Yet, in the context of the wide range of changes in health care, it is and will continue to be important for the clinician to measure and capture improvement in each individual patient for every progress report sent to third-party payers. We will always be in a situation where we must justify continued treatment to achieve optimal outcomes for our patients.

\section{Compliance with Ethics Guidelines}

Conflict of Interest J.A. Logemann has NIH funding regarding swallowing in Alzheimer's disease and industry funding from Bracco Barium to study pill swallowing as well as funding from Amedysis to examine the speech-language pathologist's role in reducing the return to acute care. She is also funded by the Paul Ruby Foundation and the ASHFoundation for investigations of Parkinson's disease related dysphagia. She is President of the CSDRG, a clinical trial group developing clinical trials in speech and hearing. She is the author of Evaluation and Treatment of Swallowing Disorders and Manual for the Videofluorographic Study of Swallowing published by PRO-ED. She has been an invited speaker for various health care related field conferences. L.L. Pitts has American Heart Association and ASHFoundation funding to study acute poststroke dysphagia recovery and Parkinson's disease related dysphagia, respectively. She has been an invited participant on local and national expert panels regarding quality measures in dysphagia management.

Human and Animal Rights and Informed Consent This article does not contain any studies with human or animal subjects performed by any of the authors.

\section{References}

Papers of particular interest, published recently, have been highlighted as:

- Of importance

•• Of major importance

1. - Logemann JA. Evaluation and treatment of swallowing disorder. Austin: PRO-ED; 1998. This book contains detailed information on normal and abnormal swallowing, and evaluation and treatment procedures in patients with sudden onset disorders, progressive disorders, and disorders of aging.

2. Smithard DG, O'Neill PA, England RE, et al. The natural history of dysphagia following a stroke. Dysphagia. 1997;12:188-97.

3. Logemann JA, Gibbons P, Rademaker AW, et al. Mechanisms of recovery of swallow after supraglottic laryngectomy. J Speech Hear Res. 1994;37:965-74.

4. Meng N, Wang T, Lien I. Dysphagia in patients with brainstem stroke: incidence and outcome. Am J Phys Med Rehabil. 2000;79:170-5.

5. Galovic M, Leisi N, Muller M, et al. Lesion location predicts transient and extended risk of aspiration after supratentorial ischemic stroke. Stroke. 2013. doi:10.1161/STROKEAHA.113. 001690.

6. Barritt AW, Smithard DG. Role of cerebral cortex plasticity in the recovery of swallowing function following dysphagic stroke. Dysphagia. 2009;24:83-90.

7. Kazandjian M, Dikeman K. Guillain-Barre syndrome and disordered swallowing. Perspect Swallow Swallow Disord. 2012;21:115-20.

8. Kuhlemeier KV. Epidemiology and dysphagia. Dysphagia. 1994;9:209-17.

9. Alagiakrishnan K, Bhanji RA, Kurian M. Evaluation and management of oropharyngeal dysphagia in different types of dementia: a systematic review. Arch Gerontol Geriat. 2013;56:1-9.

10. • Logemann JA, Gensler G, Robbins JA, et al. A randomized study of three interventions for aspiration of thin liquids in patients with dementia and Parkinson's disease. J Speech Lang Hear Res. 2008;51:173-183. This is an example of a large-scale randomized clinical trial in dysphagia which randomizes patients who aspirate on thin liquids to use one of three interventions: posture change, or one of the two thickened liquids.

11. Frowen J, Hornby C, Collins M, et al. Reducing posttreatment dysphagia: support for the relationship between radiation dose to the pharyngeal constrictors and swallowing outcomes. Pract Radiat Oncol. 2013. doi:10.1016/j.prro.2012.11.009.

12. Duprez F, Madani I, De Potter B, et al. Systematic review of dose-volume correlates for structures related to late swallowing disturbances after radiotherapy and head and neck cancer. Dysphagia. 2013;28(3):337-49. 
13. Lazarus CL, Logemann JA, Kahrilas PJ, et al. Swallow recovery in an oral cancer patient following surgery, radiotherapy, and hyperthermia. Head Neck. 1994;16:259-65.

14. Van den Berg MGA, Rutten H, Rasmussen-Conrad EL, et al. Nutritional status, food intake, and dysphagia in long-term survivors with head and neck cancer treated with chemoradiotherapy: a cross-sectional study. Head Neck. 2013. doi:10.1002/hed. 23265.

15. Rofes L, Arreola V, Romea M, et al. Pathophysiology of oropharyngeal dysphagia in the frail elderly. Neurogastroent Motil. 2010;22:851-9.

16. Daggett A, Logemann JA, Rademaker A, et al. Laryngeal penetration during deglutition in normal subjects of various ages. Dysphagia. 2006;21:270-4.

17. $\bullet$ Robbins J, Coyle J, Rosenbek J, et al. Differentiation of normal and abnormal airway penetration during swallowing using the penetration-aspiration scale. Dysphagia. 1999;14:228-232. The penetration-aspiration scale can be one important outcome measure for swallowing intervention. Clinicians can record the normalcy or abnormalcy of penetration. A significant positive outcome of dysphagia intervention is normalizing penetration.

18. Nicosia MA, Hind JA, Roecker EB, et al. Age effects on the temporal evolution of isometric and swallowing pressure. J Gerontol A Biol Sci Med Sci. 2000;55:634-40.

19. - Logemann JA, Pauloski BR, Rademaker AW, et al. Temporal and biomechanical characteristics of oropharyngeal swallow in younger and older men. J Speech Lang Hear Res. 2000;43:1264-1274. This article researches data on the effects of reduced muscular reserve in hyolaryngeal elevation and anterior movement and thus on upper esophageal tract opening in older and younger normal men.

20. Logemann JA, Pauloski BR, Rademaker AW, et al. Oropharyngeal swallow in younger and older women: videofluoroscopic analysis. J Speech Lang Hear Res. 2002;45:434-45.

21. Puthucheary Z, Harridge S, Hart N. Skeletal muscle dysfunction in critical care: wasting, weakness, and rehabilitation strategies. Crit Care Med. 2010;38:S676-82.

22. O'Conner ED, Walsham J. Should we mobilize critically ill patients? A review. Crit Care Resusc. 2009;11:290-300.

23. Kayambu G, Boots R, Paratz J. Physical therapy for the critically ill in the ICU: a systematic review and meta-analysis. Crit Care Med. 2013;41:1543-54.

24. Crary MA, Carnaby GD, Groher ME. Initial psychometric assessment of a functional oral intake scale for dysphagia in stroke patients. Arch Phys Med Rehabil. 2005;86:1516-20.

25. • Colodny N. Dysphagic independent feeders' justifications for noncompliance with recommendations by a speech-language pathologist. Am J Speech Lang Pathol. 2005;14:61-70. Colodny has carefully identified the reasons that dysphagic patients who are independent feeders chose not to comply with the intervention recommendations for feeding from the swallowing therapists such as posture change or diet changes.

26. Low J, Wyles C, Wilkinson T, et al. The effect of compliance on clinical outcomes for patients with dysphagia on videofluoroscopy. Dysphagia. 2001;16:123-7.

27. Speyer R, Baijens L, Heijnen M, et al. Effects of therapy in oropharyngeal dysphagia by speech and language therapists: a systematic review. Dysphagia. 2009;25:40-65.

28. Martino R, Foley N, Bhogal S, et al. Dysphagia after stroke: incidence, diagnosis, and pulmonary complications. Stroke. 2005;36:2756-63.

29. Wilson RD, Howe EC. A cost-effectiveness analysis of screening methods for dysphagia after stroke. PM\&R. 2012;4:273-82.

30. Logemann JA, Rademaker AW, Pauloski BR, et al. Effects of postural change on aspiration in head and neck surgical patients. Otolaryngol Head Neck. 1994;110:222-7.

31. Logemann JA, Pauloski BR, Colangelo L, et al. Effects of a sour bolus on oropharyngeal swallowing measures in patients with neurogenic dysphagia. J Speech Hear Res. 1995;38:556-63.

32. Logemann J, Kahrilas P, Kobara M, et al. The benefit of head rotation on pharyngoesophageal dysphagia. Arch Phys Med Rehabil. 1989;70:767-71.

33. Robbins JA, Gensler G, Hind J, et al. Comparison of two interventions for liquid aspiration on pneumonia incidence: a randomized controlled trial. Ann Intern Med. 2008;148:509-18.

34. Aviv JE, Kim T, Thomson JE, et al. Fiberoptic endoscopic evaluation of swallowing with sensory testing (FEESST) in healthy controls. Dysphagia. 1998;13:87-92.

35. Amin MR, Achaltis S, Lazarus CL, et al. Dynamic magnetic resonance imaging of the pharynx during deglutition. Ann Otol Rhinol Laryngol. 2013;122:145-50.

36. Institute of Medicine Committee on Quality of Health Care in America. Crossing the quality chasm: a new health system for the 21st century. National Academy Press: Washington; 2001.

37. McMicken BL, Muzzy CL. Functional outcomes of standard dysphagia treatment in first time documented stroke patients. Disabil Rehabil. 2009;31:806-17.

38. Cramer SC. Repairing the human brain after stroke: I. Mechanisms of spontaneous recovery. Ann Neurol. 2008;63:272-87.

39. Logemann JA, Baum HM, Robbins J. Conducting clinical trials in a constantly changing health care environment. J Ambul Care Manage. 2001;24:60-8. 